\title{
Atypical choroid plexus papilloma: clinical experience in the CPT-SIOP-2000 study
}

\author{
Brigitte Wrede · Martin Hasselblatt • Ove Peters · Peter F. Thall • \\ Tezer Kutluk · Albert Moghrabi · Anita Mahajan - Stefan Rutkowski · \\ Blanca Diez · Xuemei Wang · Torsten Pietsch · Rolf-Dieter Kortmann • \\ Werner Paulus · Astrid Jeibmann · Johannes E. A. Wolff
}

Received: 5 May 2009/Accepted: 1 June 2009/Published online: 20 June 2009

(C) Springer Science+Business Media, LLC. 2009

\begin{abstract}
Atypical choroid plexus papilloma (APP) represents a novel intermediate-grade subtype of choroid plexus tumor (CPT), the clinical outcome of which has not been described yet. We present the first analysis of a group of APP patients enrolled in the ongoing CPT-SIOP-2000 study of CPTs. A worldwide registration and a randomized trial for those patients who require chemotherapy started in 2000. For APP, maximal surgical resection was recommended. After surgery, patients who had undergone complete resection were observed, whereas patients with
\end{abstract}

\footnotetext{
B. Wrede · O. Peters · J. E. A. Wolff

Department of Pediatric Oncology, St. Hedwig Children's

Hospital, University of Regensburg, Regensburg, Germany

B. Wrede

e-mail: Brigitte.Wrede@barmherzige-regensburg.de

O. Peters

e-mail: ove.peters@barmherzige-regensburg.de

M. Hasselblatt - W. Paulus - A. Jeibmann

Institute of Neuropathology, University Hospital Münster,

Münster, Germany

M. Hasselblatt

e-mail: Martin.Hasselblatt@ukmuenster.de

W. Paulus

e-mail:Werner.Paulus@ukmuenster.de

A. Jeibmann

e-mail: Astrid.Jeibmann@ukmuenster.de

P. F. Thall $\cdot$ X. Wang $\cdot$ J. E. A. Wolff

Department of Biostatistics, The University of Texas M. D.

Anderson Cancer Center, Houston, TX, USA

P. F. Thall

e-mail: rex@mdanderson.org

X. Wang

e-mail: xwang1@odin.mdacc.tmc.edu
}

incompletely resected or metastasized APP were treated with six chemotherapy courses (etoposide and vincristine, combined with either carboplatin or cyclophosphamide). Risk-adapted radiotherapy was given only to patients older than 3 years of age. Of the 106 patients with a centrally confirmed CPT histology, 30 had APP, 42 CPP and 34 CPC. APP patients were significantly younger (median $=0.7$ years) than patients with CPP or CPC (both medians $=2.3$ years). Complete resection was achieved in $68(64 \%)$ patients (79\% in CPP, 63\% in APP, and $47 \%$ in

\section{T. Kutluk}

Institute of Oncology, Hacettepe University, Ankara, Turkey e-mail: tkutluk@tr.net

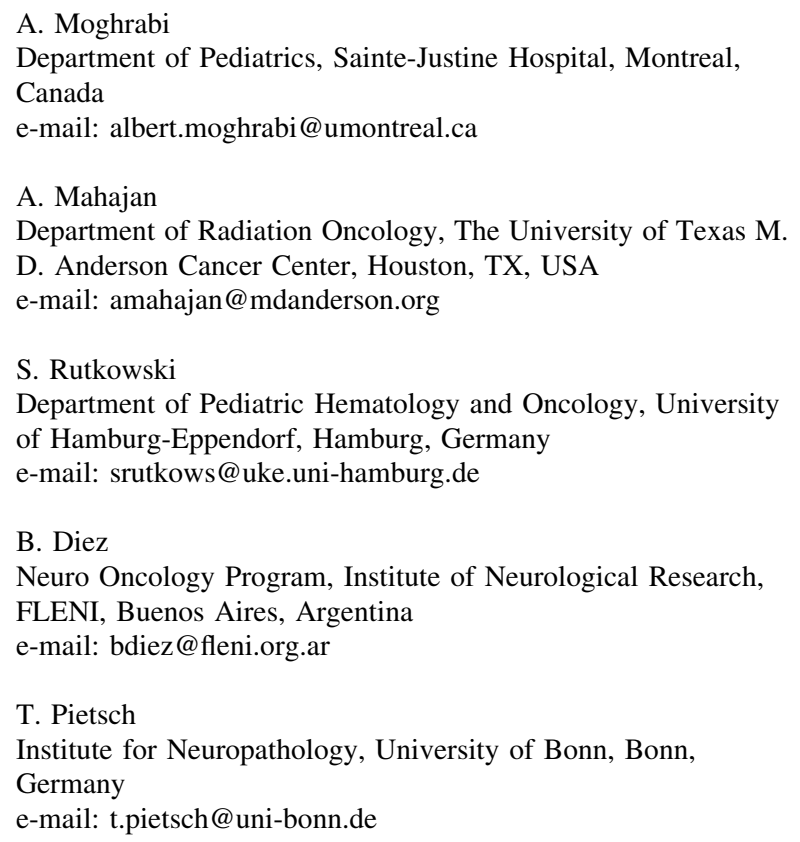


CPC). Metastases were present at diagnosis in $17 \%$ of APP patients, $5 \%$ of CPP patients, and $21 \%$ of CPC patients. All nine APP patients who received postoperative chemotherapy showed an early response after two cycles: two had complete remission, four had partial response, and three had stable disease. In the observation group of 15 patients, one event was seen, and all patients were alive. In the treatment group, one patient with a metastasized tumor and incompletely resected APP died. While APP was defined histologically, median percentages of both the Ki-67/MIB1 proliferation marker and the p53 tumor suppressor protein increased across the three histological subtypes (from CPP to APP and then CPC), suggesting that the subtypes comprise an ordinal categorization of increasingly severe CPT tumors. This ordering was reiterated by clinical outcome in the 92 patients treated per the study protocol, with 5 -year EFS rates of $92 \%$ in 39 CPP patients, $83 \%$ in 24 APP patients, and $28 \%$ in 29 CPC patients. A similar ordering was seen when all 106 patients were evaluated for EFS. APP responded favorably to chemotherapy. The intermediate position of APP between CPP and CPC was supported by the clinical data.

Keywords Choroid plexus tumors ·

Atypical choroid plexus papilloma - Survival .

Chemotherapy

\section{Introduction}

Choroid plexus tumors (CPT) are rare central nervous system neoplasms originating from the choroid plexus epithelium and therefore typically located in the ventricles (Fig. 1). They account for $1-4 \%$ of all pediatric brain tumors but represent $13 \%$ of pediatric brain tumors that occur in the first year of life [1-11]. These tumors were traditionally classified by the World Health Organization (WHO) on the basis of histological criteria as either benign choroid plexus papilloma (CPP, WHO grade I) or malignant choroid plexus carcinoma (CPC, WHO grade III) with frank signs of malignancy including brisk mitotic activity, nuclear pleomorphism, high cellularity, blurring of the papillary growth pattern, necrosis, and often diffuse brain

\section{R.-D. Kortmann \\ Department of Radiotherapy, University of Leipzig, Leipzig, Germany \\ e-mail: Rolf-Dieter.Kortmann@medizin.uni-leipzig.de}

\section{J. E. A. Wolff (ه)}

Department of Pediatrics, Unit 87, The University of Texas M. D.

Anderson Cancer Center, 1515 Holcombe Blvd., Houston, TX

77030, USA

e-mail: jwolff@mdanderson.org

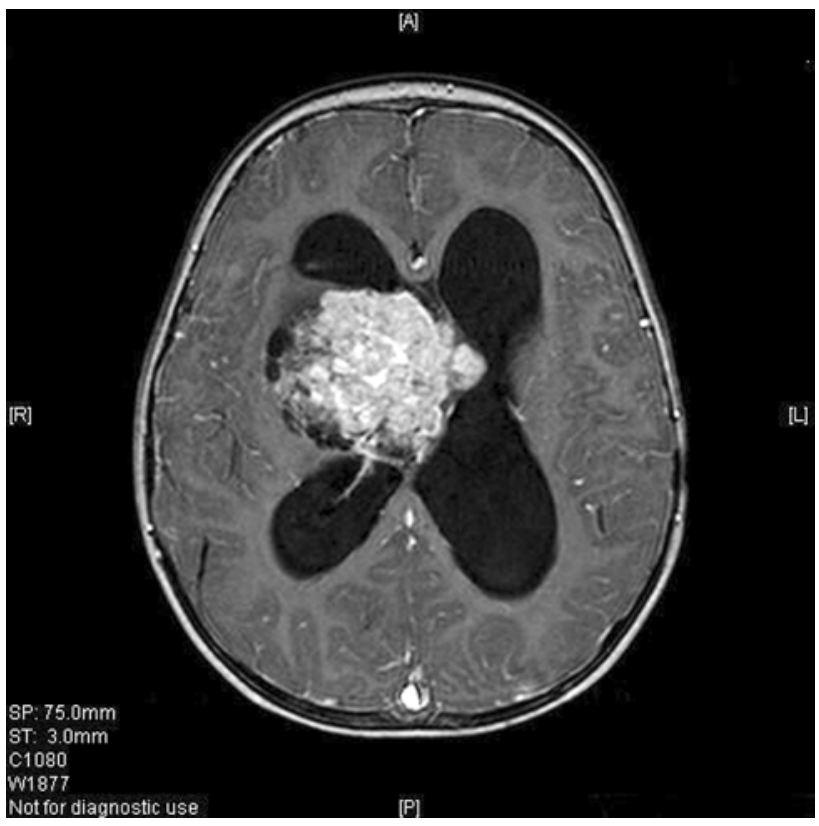

Fig. 1 Gadolinium-enhanced MRI (T1 weighted sequence) of a 2year-old girl with an atypical choroid plexus papilloma in the right lateral ventricle, with typical strong contrast enhancement of the tumor. MRI cannot be used to distinguish between the choroid plexus tumor histologic subtypes

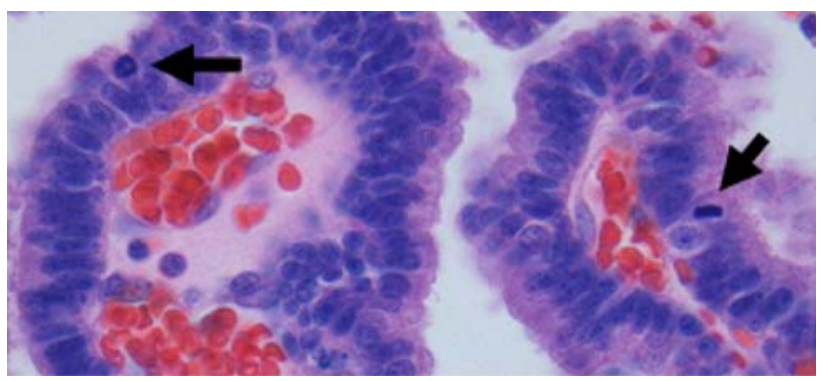

Fig. 2 Typical histological picture (hematoxylin and eosin staining) of an atypical choroid plexus papilloma with mitoses (arrows)

invasion [4]. Distinguishing between these two histologies has been difficult in some cases and prompted the coining of the term atypical papilloma (Fig. 2) to describe those tumors with an intermediate histology. In 2006 Jeibmann et al. [12] reported that mitotic activity was the only atypical histological feature independently associated with recurrence based on a retrospective series of CPP patients. Based on these data, atypical choroid plexus papilloma (APP) was defined in terms of mitotic activity (Fig. 2) and classified as grade II by the WHO in 2007 [13].

The first clinical trial specific for CPTs was begun in 2000 under the guidance of the International Society of Pediatric Oncology (SIOP). This trial (CPT-SIOP-2000) had a two-fold purpose: to create a worldwide registry of CPTs and to gain information about the clinical effectiveness of their treatment in a randomized prospective 
trial. The design of the study included the possibility of classifying a tumor as APP and allowed for modified treatment recommendations according to this diagnosis that predated the later recognition by the WHO classification committee of APP as a separate tumor subtype. After maximal surgical resection, patients with CPP or completely resected APP without metastases were observed, whereas all patients with CPC, metastases, or incompletely resected APP were treated with chemotherapy consisting of etoposide and vincristine combined with either carboplatin or cyclophosphamide. After the second course of chemotherapy, patients older than 3 years were irradiated.

After the new APP definition was published, the histology of tumors registered in the study was re-evaluated. In this paper, we present for the first time the clinical features and outcomes of 30 patients who had APP according to the new WHO definition and who were enrolled in the CPT-SIOP-2000 study.

\section{Methods}

The ongoing international CPT-SIOP-2000 study is a combination of a registry for children and adults with CPTs and a randomized investigator-initiated clinical treatment trial for patients requiring postoperative treatment. The treatment trial has been reviewed and approved by the brain tumor subcommittee of SIOP and is registered at Clinicaltrials.gov as NCT00500890. All patients with newly diagnosed and histologically confirmed CPTs are registered, irrespective of histological tumor subtype, the extent of the tumor, or age at diagnosis. Patients with CPP and completely resected APP are then observed, whereas all patients with CPC, metastatic disease or incompletely resected APP are treated with 6 cycles of chemotherapy. The chemotherapy regimen consists of etoposide $100 \mathrm{mg} / \mathrm{m}^{2}$ administered daily for 5 days, vincristine $1.5 \mathrm{mg} / \mathrm{m}^{2}$ on day 5 , and a randomization to either carboplatin $350 \mathrm{mg} / \mathrm{m}^{2}$ administered daily for 2 days or cyclophosphamide $1 \mathrm{~g} / \mathrm{m}^{2}$ administered daily for 2 days. Radiotherapy is given to patients older than 3 years of age after the second cycle of chemotherapy. The radiation field is stratified by the expected prognosis. Craniospinal irradiation with 35.2 Gy given in 22 1.6-Gy fractions and a local boost up to a total of $54 \mathrm{~Gy}$ is given to patients with CPC nonresponsive to chemotherapy (stable disease or progressive disease) and those with metastasized CPC and APP. Local radiation with 54 Gy administered in 30 1.8-Gy fractions is given to the others.

\section{Participants}

Patients analyzed in this interim report were all registered in the CPT-SIOP-2000 study. Informed consent was obtained prospectively from patients, parents, or legal guardians in accordance with national laws and with the local guidelines of the participating centers, and this analysis is in keeping with the original goal of the study described in the consent forms to learn more about choroid plexus tumors was fulfilled by this analysis.

\section{Clinical assessment}

The extent of disease in all patients was evaluated by brain and spine MRI with and without gadolinium. Complete resection was defined according to the SIOP guidelines [14]. If the neurosurgeon noted tumor adhesions or residual gross disease, the resection was classified as partial despite the absence of visible tumor on postoperative imaging. MRI was performed after the second and sixth cycles of chemotherapy to determine response (early response, best response) and in the case of an initial partial resection to determine if a second surgery was needed. Response was categorized as disease progression, greater than $25 \%$ progression of the product of the longest tumor diameter and its perpendicular determined by MRI, irrespective of clinical signs; complete remission, a $100 \%$ decrease of the postoperative cross-sectional tumor area, in case of only malignant pleocytosis the disappearance of tumor cells from cerebrospinal fluid; or partial response, a decrease of at least $50 \%$ of the postoperative cross-sectional tumor area. Continuous complete remission (CCR) was defined as no evidence of disease on MRI after surgery and during follow-up. Diagnostic lumbar puncture was done at the time of diagnosis, before or 14 days after initial surgery.

\section{Histology}

A central review of pathology was not required for CPTSIOP-2000 study entry. However, since previous interim analyses had shown an agreement of only $70 \%$ between local pathology reports and reference reports only those patients with central review and confirmed diagnosis of CPT at the time of this analysis were included in this analysis. All APP specimens were reviewed by the reference neuropathologist according to the current WHO criteria [13].

Immunohistochemical staining was performed for the proliferation marker Ki-67/MIB-1, for tumor suppressor p53 protein, and hSNF5/INI1. Following deparaffinization and boiling, $2-\mu \mathrm{m}$ sections were stained using monoclonal antibodies directed against Ki-67 (MIB-1; 1:100, citrate buffer pH 6 for antigen retrieval; Dako, Glostrup, Denmark), p53 (BP53-12; 1:200, citrate buffer pH 6 for antigen retrieval; Novocastra, Leica Microsystems, Wetzlar, Germany) and hSNF5/INI1 (BAF47; 1:200, citrate buffer pH 9.9 for antigen retrieval; BD Biosciences, San Jose, $\mathrm{CA}$ ) and the $\mathrm{ABC}$ method on an automated staining system 
(TechMate, Dako). The number of tumor cells positive for Ki-67/MIB-1 and p53 was quantified by counting 2,000 tumor cell nuclei.

\section{Statistical methods}

Patient characteristics were summarized using median and range for continuous variables and frequency and percentage for categorical variables. Associations between histology and continuous variables were detected by the KruskalWallis test [15] and associations between histology and categorical variables by the generalized Fisher's exact test [16]. Box plots were used to illustrate differences in Ki-67/ MIB-1 percentage between histology groups. Overall survival (OS) time was defined as the time from the date of diagnosis to the date of death. Event-free survival (EFS) time was defined as the time from the date of diagnosis to the date of death or relapse, whichever occurred first. Unadjusted OS and EFS probabilities from diagnosis to the time of this analysis were estimated using the Kaplan-Meier method [17] and were compared between subgroups of patients using the log-rank test [18]. The Cox proportional hazards regression model [19] was used to assess the effects of patient characteristics and treatment on OS and EFS, with preliminary goodness of fit assessed by the GrambschTherneau test and martingale residual plots [20]. Because of the colinearity among several covariates, to avoid unstable Cox model fits, separate Cox models were fit so that collinear covariates did not appear in the same model. All computations were carried out in S-PLUS [21].

\section{Results}

\section{Patient characteristics}

From January 2000 to June 2008, 133 patients from 21 nations were registered in the CPT-SIOP-2000 study with an initial diagnosis of CPT. Central review was available for 116 patients. Of those, 10 patients were excluded from the present analysis due to histology other than a CPT (e.g. atypical teratoid rhabdoid tumor, medulloepithelioma and papillary tumors of the pineal region). Thus, 106 patients (54 male, 52 female) with histology confirmed as CPT were potentially eligible for the current analysis. Of those, 30 patients had APP and were selected for this review, 42 had CPP, and 34 had CPC.

The 30 patients with APP had a median age at diagnosis of 0.7 years and were significantly younger than patients with CPP or with CPC (median of 2.3 years for both; $P=0.003$, Kruskal-Wallis test). Gender was almost equally distributed within each subtype, with males comprising $50 \%$ of CPC and APP patients and $52 \%$ of CPP patients. Primary tumors were located in the lateral ventricles in $83 \%$ of patients with APP, $71 \%$ of patients with $\mathrm{CPP}$, and $88 \%$ of patients with CPC. Only $3 \%$ of tumors were found in the fourth ventricle in APP patients, versus $19 \%$ in CPP patients and $12 \%$ in CPC patients. The remaining $13 \%$ of APP patients had tumors located in the third ventricle (10\% in CPP and $0 \%$ in CPC). Complete resection was achieved in 33 of the 42 (79\%) CPP patients, 19 of the $30(63 \%)$ APP patients, and 16 of the $34(47 \%)$ CPC patients. Metastases from the primary tumor were noted in $5(17 \%)$ APP patients, almost as often as in the CPC group ( $n=7,21 \%)$, while the rate was very low $(n=2,5 \%)$ in the CPP group (Table 1).

\section{Immunohistochemistry}

Material sufficient for immunohistochemical staining for Ki67/MIB-1, p53 protein, and hSNF5/INI1 was available for 73 cases (32 CPP, 24 APP, and 17 CPC). As shown in Table 1 and Fig. 3a, the respective median $\mathrm{Ki}-67 / \mathrm{MIB}-1$ proliferation indices were 1.3, 9.1, and 20.3 in the CPP, APP, and CPC histology groups, respectively $(P<0.0001$, Wilcoxon rank sum test). Similarly, as shown in Table 1 and Fig. $3 b$, the numbers and percentages of patients with tumors exhibiting p53-positive nuclei were zero of 31 in CPP patients, two of $21(9.5 \%)$ in APP patients, and eight of $17(47.1 \%)$ in CPC patients $(P<0.0001$, generalized Fisher's exact test). All CPTs examined expressed nuclear hSNF5/INI1.

Response to chemotherapy

Of 106 patients, 92 (24 patients with APP, 39 with CPP, and 29 with CPC) were treated according to the CPTSIOP-2000 study protocol. The 14 patients not treated per protocol were thus excluded from further analysis here mainly because they received either a chemotherapy regimen or a therapeutic strategy not matching that of the study protocol. Of 24 patients with APP, 15 (62.5\%) had a complete resection and no metastases; therefore, per protocol they were followed up with surveillance only. With a mean follow-up time of 1.7 years, one event was seen in this group in a 1.8-year-old boy originally diagnosed with a right lateral ventricular APP. At 2.1 years of follow-up, he was found to have local recurrence and disseminated disease with multiple nodules in the brain and the spine. He was then treated according to the CPT-SIOP-2000 protocol and received six cycles of chemotherapy and craniospinal irradiation, which resulted in a stable disease. At the time of the interim analysis, he was 4.6 years old and had experienced no further relapse.

Nine patients with APP received initial postoperative chemotherapy, and all responded. Four who had no metastases at diagnosis were known to have grossly evident 
Table 1 Characteristics of the CPT-SIOP-2000 study patients

\begin{tabular}{|c|c|c|c|c|}
\hline & CPP & APP & $\mathrm{CPC}$ & $P$ value \\
\hline No. patients & 42 & 30 & 34 & \\
\hline No. patients treated per protocol & 39 & 24 & 29 & \\
\hline Age [years; median (range)] & $2.3(0.02-45.6)$ & $0.7(0.1-9.2)$ & $2.3(0.3-17.1)$ & 0.003 \\
\hline Ki-67/MIB-1 [\%; median (range) $]^{\mathrm{a}}$ & $1.3(0-11.9)$ & $9.1(0.5-35.6)$ & $20.3(7.8-42.5)$ & $<0.0001$ \\
\hline P53 ${ }^{\mathrm{a}}$ absent & $31(100 \%)$ & $19(91 \%)$ & $9(53 \%)$ & $<0.0001$ \\
\hline $\mathrm{P} 53^{\mathrm{a}}$ present & $0(0 \%)$ & $2(9.5 \%)$ & $8(47 \%)$ & \\
\hline Male & $22(52 \%)$ & $15(50 \%)$ & $17(50 \%)$ & 1.00 \\
\hline Female & $20(48 \%)$ & $15(50 \%)$ & $17(50 \%)$ & \\
\hline Chemotherapy & & & & $<0.0001$ \\
\hline Yes & $6(14 \%)$ & $14(47 \%)$ & $31(91 \%)$ & \\
\hline No & $36(86 \%)$ & $16(53 \%)$ & $3(9 \%)$ & \\
\hline Location & & & & 0.06 \\
\hline Lateral ventricle & $30(71 \%)$ & $25(83 \%)$ & $30(88 \%)$ & \\
\hline Third ventricle & $4(10 \%)$ & $4(13 \%)$ & $0(0 \%)$ & \\
\hline Fourth ventricle & $8(19 \%)$ & $1(3 \%)$ & $4(12 \%)$ & \\
\hline Metastases & & & & 0.09 \\
\hline Yes & $2(5 \%)$ & $5(17 \%)$ & $7(21 \%)$ & \\
\hline No & $40(95 \%)$ & $25(83 \%)$ & $27(79 \%)$ & \\
\hline Resection & & & & 0.05 \\
\hline Complete & $33(79 \%)$ & $19(63 \%)$ & $16(47 \%)$ & \\
\hline Subtotal/partial & $8(19 \%)$ & $10(33 \%)$ & $16(47 \%)$ & \\
\hline Biopsy & $1(2 \%)$ & $1(3 \%)$ & $2(6 \%)$ & \\
\hline
\end{tabular}

$A P P$ Atypical choroid plexus papilloma, $C P C$ choroid plexus carcinoma, $C P P$ choroid plexus papilloma

${ }^{a}$ Data on Ki-67/MIB-1 and p53 were missing for 33 and 37 patients, respectively

residual tumor after surgery. Two of these four had stable disease after two cycles of chemotherapy, and both became tumor free later: one after local irradiation (54 Gy) and the other after further resection. The remaining two patients responded partially to two cycles of chemotherapy and reached complete remission after further treatment: one with local radiation $(45 \mathrm{~Gy})$ and the other with chemotherapy only. All four patients were alive and had experienced no event after a mean observation time of 3.3 years.

Five patients were treated for metastatic APP at original presentation with or without residual tumor at the primary site. Two of these five patients, classified as having R0M1 and R0M2 disease, had a complete remission after two cycles of chemotherapy. A third patient, with R0M3 disease, had a partial response to two cycles of chemotherapy and therefore received craniospinal irradiation. The fourth patient was classified as having R2M2 APP, which stabilized in early response, after two cycles of chemotherapy, but had achieved complete remission at the end of treatment (eight cycles of chemotherapy). The fifth patient, a 7.3-year-old boy with R2M3 disease at diagnosis, was found to have one of the two post-treatment events in the APP group. Two cycles of chemotherapy resulted in a partial early response. He then received craniospinal irradiation followed by a boost to the tumor bed (total dose $=54 \mathrm{~Gy}$ ); a small residual tumor was observed thereafter; it remained stable for 1.6 years. However, at 2.3 years, the patient had locally relapsed APP, which was completely resected. Afterward, the child received highdose chemotherapy with stem cell rescue, but he had a second local relapse with extensive spread 1 year later. He died with progressive disease 4.3 years after the original diagnosis.

In summary, the early response to chemotherapy of those nine APP patients receiving postsurgical treatment was complete remission in two (22\%), partial response in four $(44 \%)$, and stable disease in three $(33 \%)$. These rates of response seemed similar to those seen in patients with CPC (Fig. 4). With further treatment, an additional four of these nine patients reached complete remission, resulting in a "best response" rate of $67 \%$ (six of nine patients) for those treated.

Overall survival and event-free survival

Of the 92 patients who were treated according to the protocol, eight $(8.7 \%)$ had died at the time of this writing. The median follow-up time was 2.2 years (range $0-8.2$ years). 

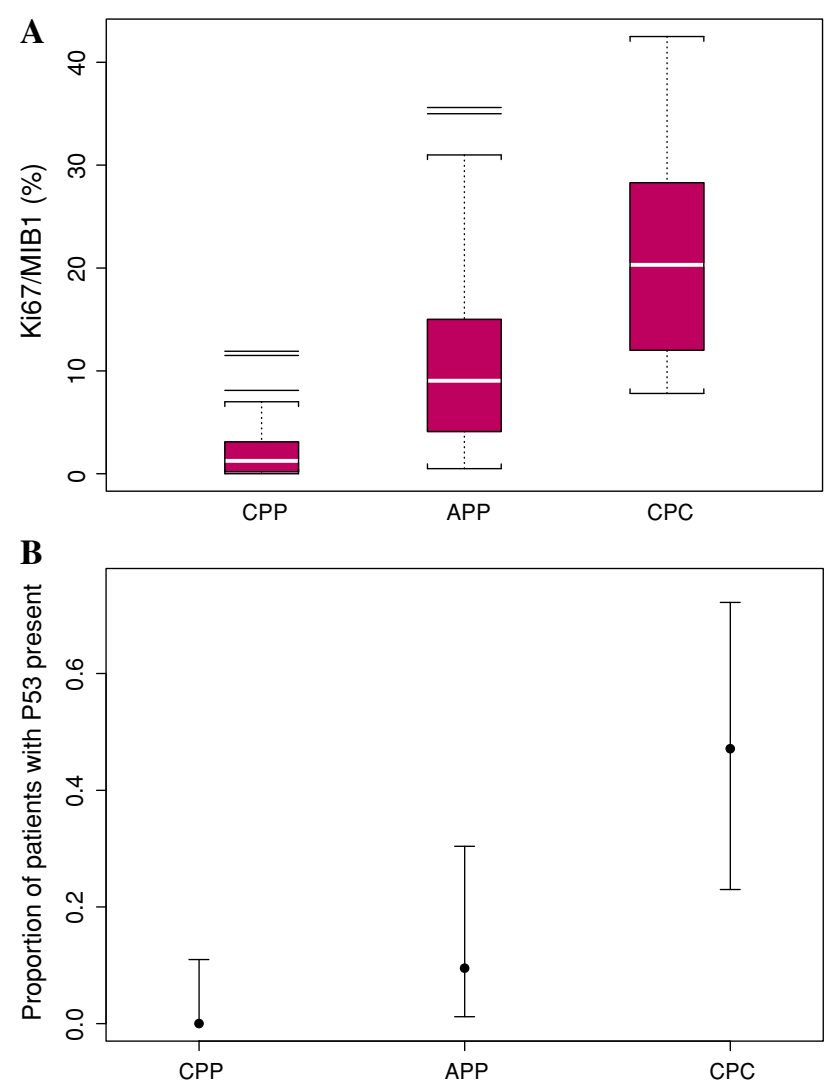

Fig. 3 a Box plot for the Ki-67/MIB-1 (\%) by histology group in 73 patients (data missing in 33 patients). Immunohistochemical staining for Ki-67/MIB-1 in choroid plexus papillomas (CPP, $n=32$ ), atypical choroid plexus papillomas (APP, $n=24$ ), and choroid plexus carcinomas (CPC, $n=17$ ) showed a significant effect of tumor grade on Ki-67/MIB-1 proliferation indices $(P<0.0001)$. b The proportions of 69 patients with p53-positive tumors by histology group (data were missing for 37 patients). The data show a significant effect of tumor grade on percentage of cases exhibiting nuclear accumulation of p53 protein $(P<0.0001)$. For each histology group, the dot represents the mean sample proportion and the lines show the exact $95 \%$ confidence interval

In comparison with the CPC and CPP patients, APP patients had intermediate OS and EFS, on average. The 5year OS probabilities were $100 \%$ in 39 CPP patients, $89 \%$ (95\% CI, 71-100\%) in 24 APP patients, and 36\% (95\% CI, $9-100 \%$ ) in 29 CPC patients (Fig. $5, P=0.0001$; log-rank test). Fifteen patients $(16.3 \%)$ had disease progression or relapse or died. Similar to OS, the 5-year EFS probabilities were $92 \%$ (95\% CI, 77-100\%) in CPP patients, $83 \%$ (95\% CI, 65-00\%) in APP patients, and 28\% (95\% CI, 7-100\%) in CPC patients (Fig. 6, $P<0.0001$; log-rank test). A similar ordering was seen when EFS was evaluated by histology for all 106 patients registered. Whether a patient received chemotherapy was highly associated with histology, with 28 of the 29 (97\%) CPC patients versus 12 of the 63 (19\%) CPP and APP patients treated per protocol receiving chemotherapy $(P<0.0001$, Fisher's exact test).

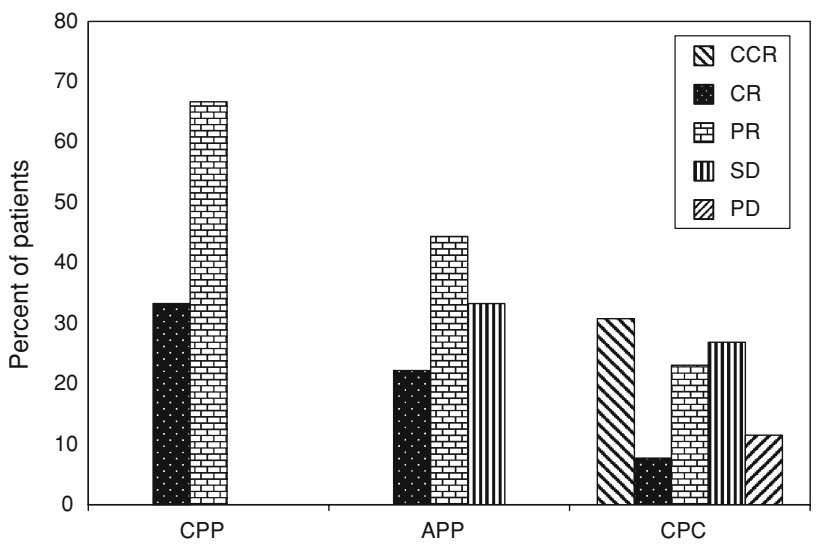

Fig. 4 Response status by histology group in 92 patients treated according to the study protocol with 2 cycles of chemotherapy. APP Atypical choroid plexus papilloma, CCR continuous complete remission (patients with complete resection and no tumor progression), $C P C$ choroid plexus carcinoma, $C P P$ choroid plexus papilloma, $C R$ complete remission (tumor disappearance on treatment), $P R$ partial response, $S D$ stable disease, $P D$ progressive disease

Table 2 Univariate Cox proportional hazards model for overall survival (total $n=92, n \_$death $=8$ )

\begin{tabular}{lllll}
\hline Variable & Coefficient & SE & HR & $P$ value \\
\hline CPC (vs. CPP/APP) & 3.27 & 1.09 & 26.4 & 0.003
\end{tabular}

$C P C$ Choroid plexus carcinoma, $C P P$ choroid plexus papilloma, $A P P$ atypical choroid plexus papilloma, $S E$ standard error, $H R$ hazard rate

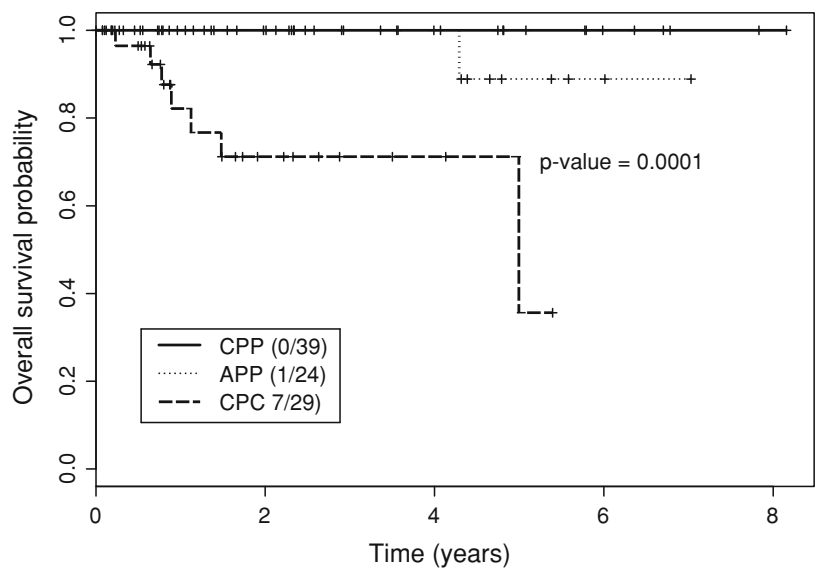

Fig. 5 Overall survival of choroid plexus tumor patients (KaplanMeier estimate). APP Atypical choroid plexus papilloma, $C P C$ choroid plexus carcinoma, $C P P$ choroid plexus papilloma. Patient data were classified as censored if the patients were alive at the end of the observation period

This difference was a consequence of the treatment algorithm. Consequently, to avoid an unstable model due to colinearity, separate univariate Cox models were fit to assess the associations between histology and OS (Table 2), between chemotherapy and EFS and between histology and EFS. These are summarized in Table 3 for histology 


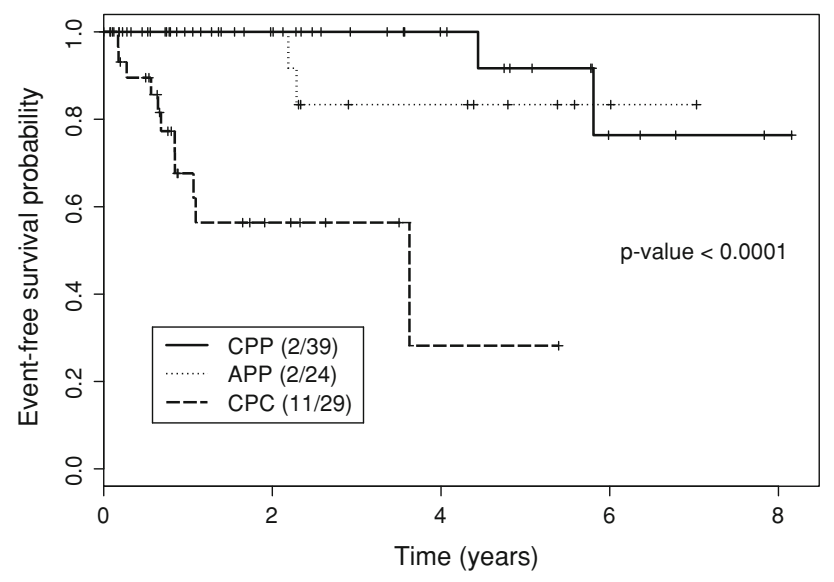

Fig. 6 Event-free survival (Kaplan-Meier estimate). APP Atypical choroid plexus papilloma, $C P C$ choroid plexus carcinoma, $C P P$ choroid plexus papilloma. Events were defined as tumor progression, tumor recurrence, or death. Patient data were censored if the patients were event free at the end of the observation period

Table 3 Univariate Cox proportional hazards model for event-free survival (total $n=92, n$ with an event $=15$ )

\begin{tabular}{lllcc}
\hline Variable & Coefficient & SE & HR & $P$ value \\
\hline CPC (vs. CPP/APP) & 2.72 & 0.68 & 15.2 & $<0.0001$ \\
Chemo = yes (vs. no)* & 1.86 & 0.65 & 6.40 & 0.004 \\
\hline
\end{tabular}

$C P C$ Choroid plexus carcinoma, $C P P$ choroid plexus papilloma, $A P P$ atypical choroid plexus papilloma, $S E$ standard error, $H R$ hazard rate

* Chemotherapy was significantly associated with histology $P<0.0001$ (Fisher's exact test)

$(P<0.0001)$ and chemotherapy $(P=0.004)$. In this regard, the large hazard ratio of 6.4 for chemotherapy versus no chemotherapy certainly should not be interpreted to mean that chemotherapy causes a higher rate of progression or death. This result is merely an artifact of the fact that, in this data set, chemotherapy is essentially a surrogate variable for the much more unfavorable CPC histology.

\section{Patients following nonprotocol treatment algorithms}

Three of the six APP patients excluded from the analyses because they were not treated according to CPT-SIOP2000 study protocol were treated with six cycles of chemotherapy despite complete resection. These patients were all alive after a mean observation time of 3.6 years had experienced no post-treatment events. Among the other three patients, one underwent a biopsy followed by two cycles of chemotherapy, and after second surgery with a gross total resection, no further treatment was given. This patient had a local recurrence after 3.2 years. The fifth patient was excluded from further analysis because the APP was found to be part of a mature teratoma on pathological analysis of the specimen. The sixth patient did not receive further treatment because of an assumed complete resection, but he was diagnosed with a local regional recurrence 0.5 years after the initial surgery and thus was classified as having had residual disease.

\section{Discussion}

APPs are rare tumors, and their clinical course is not well understood. The re-evaluation of APP cases with respect to the new diagnostic criteria [13] within the CPT-SIOP-2000 study allowed for the first time analyses of both the relevance of prognostic factors as well as the optimal treatment of these tumors. In our series, the APP group showed an intermediate position between the CPP and CPC groups in terms of Ki-67/MIB-1 proliferation indices, percentages of patients with tumor exhibiting p53-positive nuclei, and 5year EFS rates. This ordering of histologies (CPP, APP, and CPC) by increasing severity also was reiterated by the increasing percentages of patients with metastases and the decreasing complete resection rates across these three CPT subtypes. The high rate of metastases in the group of patients with APP, which was close to that of patients with $\mathrm{CPC}$, is remarkable. Several case reports showed that CPP can also metastasize with diverse outcomes [22-25], although those metastases have mostly been found at the time of relapse. One notable exception to the above ordering was age: The APP patients were younger than expected. However, because it is generally believed that higher mitosis rates are more often detected in younger patients, it cannot be ruled out that the current APP definition is biased toward younger CPT patients, thereby explaining the younger age of the APP group. An alternative explanation is that the present APP definition includes tumors with different biologic features specific to very young patients, although the immunohistochemical staining results described here do not support such a hypothesis.

Whereas Ki-67/MIB-1 labeling indices in CPPs were lower than those in our CPCs but comparable to those in previous small series [26, 27], our data demonstrate that proliferative activity in APPs is intermediate between CPPs and CPCs. Since Ki-67/MIB-1 labeling can be more readily quantified as compared to mitotic activity, Ki-67/MIB-1 labeling might once well contribute toward a better definition of APP, as has been achieved in other entities such as atypical central neurocytoma [28]. Determination of a cut-off Ki-67/MIB-1 value that predicts a higher risk of recurrence would be most desirable, but determining such a value is not yet feasible because of the relatively short follow-up of patients in the ongoing CPT-SIOP-2000 study. One report showed tumor polyploidy with a gain of 
genetic material of almost all chromosomes in an 16-yearold boy with APP [29]. In our series, in contrast with CPCs, APPs only rarely stained positive for p53, suggesting that, as in CPP [26], genetic alterations of TP53 resulting in nuclear accumulation of p53 protein are rare in APPs. The finding of retained nuclear hSNF5/INI1 staining in not only APPs but also in all the CPPs and CPCs examined extends previous observations [30-32] and argues strongly against a role for an inactivating mutation of the SMARCBl gene in CPTs.

Treatment of CPTs typically includes some combination of the classical modalities of surgery, radiotherapy, and chemotherapy. The therapeutic algorithm determining the intensity, timing, and order of how they are used is quite complex, but fundamentally it depends on the patient's age, the extent of disease, the tumor histology, and the outcome at each stage of therapy. The CPT-SIOP-2000 study included the response to the initial chemotherapy in the algorithm. The data shown here provide an overall assessment of how well this algorithm has performed, in particular within the newly defined subgroup of APP patients. Gross total tumor resection is considered as the first therapeutic approach and also the most important prognostic factor in all CPTs [9, 33-38]. In CPP patients, complete resection alone is thought to be curative $[9,36$, 37, 39]. The experience of the CPT-SIOP-2000 study does not completely confirm this opinion. Relapses were seen even in the CPP group, including one patient whose tumor histology transformed to CPC [40]. Some APP patients treated according to the protocol experienced events after complete resection, as did a patient treated according to a different algorithm. Since relapse often occurs after many years [24, 39, 41], our results suggest the need for close follow-up and suggest that a second surgery should be performed in the case of recurrence.

Chemotherapy for CPC is well established [38, 42]. However, information about the role of chemotherapy in CPP or APP is limited and primarily based on its use in patients with relapsed tumors. Several reports have shown response of recurrent or metastatic CPP to various regimens of chemotherapy [43-46], and one report about a CPP with focal regions of high mitotic activity, resembling an APP by the new definition, showed more than $50 \%$ tumor regression after chemotherapy [43]. In our study, we were able to demonstrate for the first time in a large study that APP tumors are chemosensitive (Fig. 4), with all nine patients responding to chemotherapy.

Craniospinal irradiation was usually used in this study, and in combination with chemotherapy it resulted in tumor control in most of the APP patients with metastatic disease. Although this is by far the largest series of APP patients ever systematically analyzed, patient numbers are still too small to assess the prognostic value of or best treatment for metastatic APP. Because of the risk of late neurological sequelae, only a few patients may receive radiotherapy. However, in a greater context, patients were seen to have a response to radiotherapy, and the overall good EFS and OS support the inclusion of radiation as part of the definitive approach in selected patients with APP. This view is supported by single reports of others [39, 47] and by earlier results from a meta analysis [48]. Some authors have recommended resecting metastases or have reported successful resection of metastases [25, 49, 50], but it is questionable whether surgical therapy of metastases without adjuvant therapy is sufficient for tumor control, especially after leptomeningeal seeding has occurred [51]. The survival of patients with APP in this analysis was good and only slightly inferior to that of CPP patients. However, in contrast to CPP, many APP tumors had metastases or were subtotally resected, and adjuvant treatment was necessary. Given these poor prognostic features, we believe that the histology-specific treatment algorithm led to the excellent outcomes. Moreover, we disagree with the notion that APP and CPP can be viewed as diseases to be treated similarly [9, 36, 37, 39]. The aggressive course of APP, as seen in one patient who died due to tumor progression and another whose APP relapsed after he/she did not receive additional treatment, supports this view. Furthermore, malignant progression of APP to CPC has been described [40], also indicating the higher malignant potential of the disease.

In conclusion, the newly defined WHO grade II APP histology has both prognostic features and clinical outcome rates intermediate between those of CPP and CPC, although our APP patients were younger and had more metastases than expected. Further studies are necessary to evaluate the relevance of these findings. The response to chemotherapy was high, and OS in APP patients treated according to the CPT-SIOP-2000 protocol was almost as good as that of CPP patients who did not receive treatment. This analysis suggests that chemotherapy and radiotherapy are of value in APP patients with incompletely resected tumors, metastatic disease or recurrent tumor.

Acknowledgements The CPT-SIOP-2000 study was designed in 1999, and far more people had significantly more academic impact during the past decade than suggested by the list of authors. We thank David Ellison (Pathology, Memphis, TN, USA) for excellent neuropathology work and helpful scientific discussion. We thank Richard Sposto (Children's Hospital of Los Angeles, USA) for statistical support and enthusiasm to get the study. Among the numerous clinical participants, we wish to thank R. Corbett, M. Nathrath, S. Van Gool, L. Leskova, G. Ebetsberger, J. Sterba, N. Jorch, D. Ashley, B. Erdlenbruch, C. Kramm, J. Finlay, M. Warmuth Metz, R. Kebudi, T. Hassall, M. Dittrich, N. Konoplya, M. v. d. Hagen, I. Slavc, U. Kontny, M. Albert, R. Reddingius, D. C. Kiss, A. Navajos, M. L. Garre, and many others. The CPT-SIOP-2000 study is supported by the Deutsche Kinderkrebsstiftung, also supporting B. W. M. H. and W. P. are supported by Deutsche Krebshilfe (Grant 108263). 


\section{References}

1. Galassi E, Godano U, Cavallo M, Donati R, Nasi MT (1989) Intracranial tumors during the 1st year of life. Childs Nerv Syst 5:288-298

2. Duffner PK, Kun LE, Burger PC, Horowitz ME, Cohen ME, Sanford RA, Krischer JP, Mulhern RK, James HE, Rekate HL et al (1995) Postoperative chemotherapy and delayed radiation in infants and very young children with choroid plexus carcinomas. The Pediatric Oncology Group. Pediatr Neurosurg 22:189-196

3. St Clair SK, Humphreys RP, Pillay PK, Hoffman HJ, Blaser SI, Becker LE (1991) Current management of choroid plexus carcinoma in children. Pediatr Neurosurg 17:225-233

4. Rickert CH, Paulus W (2001) Tumors of the choroid plexus. Microsc Res Tech 52:104-111

5. Yadav S, Dubey AP (1993) Choroid plexus papilloma in infancy. Indian Pediatr 30:1217-1218

6. Hashizume A, Kodama Y, Hotta T, Yuki K, Taniguchi E, Eguchi K, Yamasaki F, Katayama S, Yamane T, Hada Y (1995) Choroid plexus carcinoma in the lateral ventricle-case report. Neurol Med Chir (Tokyo) 35:742-744

7. Johnson DL (1989) Management of choroid plexus tumors in children. Pediatr Neurosci 15:195-206

8. Laurence KM (1979) The biology of choroid plexus papilloma in infancy and childhood. Acta Neurochir (Wien) 50:79-90

9. McGirr SJ, Ebersold MJ, Scheithauer BW, Quast LM, Shaw EG (1988) Choroid plexus papillomas: long-term follow-up results in a surgically treated series. J Neurosurg 69:843-849

10. Wrede B, Liu P, Ater J, Wolff JE (2005) Second surgery and the prognosis of choroid plexus carcinoma-results of a meta-analysis of individual cases. Anticancer Res 25:4429-4433

11. Wrede B, Liu P, Wolff JE (2007) Chemotherapy improves the survival of patients with choroid plexus carcinoma: a metaanalysis of individual cases with choroid plexus tumors. J Neurooncol 85:345-351

12. Jeibmann A, Hasselblatt M, Gerss J, Wrede B, Egensperger R, Beschorner R, Hans VH, Rickert CH, Wolff JE, Paulus W (2006) Prognostic implications of atypical histologic features in choroid plexus papilloma. J Neuropathol Exp Neurol 65:1069-1073

13. Louis D, Ohgaki H, Wiestler O, Cavenee W, Burger P, Jouvet A, Scheithauer B, Kleihues P (2007) The 2007 WHO classification of tumours of the central nervous system. Acta Neuropathol 114:97-109

14. Gnekow AK (1995) Recommendations of the Brain Tumor Subcommittee for the reporting of trials. SIOP Brain Tumor Subcommittee. International Society of Pediatric Oncology. Med Pediatr Oncol 24:104-108

15. Hollander M, Wolfe DA (1973) Nonparametric statistical methods. Wiley, New York

16. Fisher RA (1922) On the interpretation of $\chi^{2}$ from contingency tables, and the calculation of P. J R Stat Soc 85:87-94

17. Kaplan EL, Meier P (1958) Nonparametric estimator from incomplete observations. J Am Stat Assoc 53:457-481

18. Mantel N (1966) Evaluation of survival data and two new rank order statistics arising in its consideration. Cancer Chemother Rep 50:163-170

19. Cox DR (1972) Regression models and life tables (with discussion). J R Stat Soc 34:187-220

20. Therneau TM (2000) Grambsch: modeling survival data. Springer, New York

21. Venables WN, Ripley BD (1999) Modern applied statistics with Splus. Springer, New York

22. Valladares JB, Perry RH, Kalbag RM (1980) Malignant choroid plexus papilloma with extraneural metastasis. Case report. J Neurosurg 52:251-255
23. Leblanc R, Bekhor S, Melanson D, Carpenter S (1998) Diffuse craniospinal seeding from a benign fourth ventricle choroid plexus papilloma. Case report. J Neurosurg 88:757-760

24. Yu H, Yao TL, Spooner J, Stumph JR, Hester R, Konrad PE (2006) Delayed occurrence of multiple spinal drop metastases from a posterior fossa choroid plexus papilloma. Case report. J Neurosurg Spine 4:494-496

25. Uff CE, Galloway M, Bradford R (2007) Metastatic atypical choroid plexus papilloma: a case report. J Neurooncol 82:69-74

26. Carlotti CG Jr, Salhia B, Weitzman S, Greenberg M, Dirks PB, Mason W, Becker LE, Rutka JT (2002) Evaluation of proliferative index and cell cycle protein expression in choroid plexus tumors in children. Acta Neuropathol 103:1-10

27. Vajtai I, Varga Z, Aguzzi A (1996) MIB-1 immunoreactivity reveals different labelling in low-grade and in malignant epithelial neoplasms of the choroid plexus. Histopathology 29:147-151

28. Soylemezoglu F, Scheithauer BW, Esteve J, Kleihues P (1997) Atypical central neurocytoma. J Neuropathol Exp Neurol 56:551-556

29. Brassesco MS, Valera ET, Neder L, Castro-Gamero AM, Arruda D, Machado HR, Sakamoto-Hojo ET, Tone LG (2008) Polyploidy in atypical grade II choroid plexus papilloma of the posterior fossa. Neuropathology. doi:10.1111/j.1440-1789.2008. 00949.x

30. Hasselblatt M, Bohm C, Tatenhorst L, Dinh V, Newrzella D, Keyvani K, Jeibmann A, Buerger H, Rickert CH, Paulus W (2006) Identification of novel diagnostic markers for choroid plexus tumors: a microarray-based approach. Am J Surg Pathol 30:66-74

31. Judkins AR, Burger PC, Hamilton RL, Kleinschmidt-DeMasters B, Perry A, Pomeroy SL, Rosenblum MK, Yachnis AT, Zhou H, Rorke LB, Biegel JA (2005) INI1 protein expression distinguishes atypical teratoid/rhabdoid tumor from choroid plexus carcinoma. J Neuropathol Exp Neurol 64:391-397

32. Mueller W, Eum JH, Lass U, Paulus W, Sarkar C, Bruck W, von Deimling A (2004) No evidence of hSNF5/INI1 point mutations in choroid plexus papilloma. Neuropathol Appl Neurobiol 30:304-307

33. Ellenbogen RG, Winston KR, Kupsky WJ (1989) Tumors of the choroid plexus in children. Neurosurgery 25:327-335

34. Packer RJ, Perilongo G, Johnson D, Sutton LN, Vezina G, Zimmerman RA, Ryan J, Reaman G, Schut L (1992) Choroid plexus carcinoma of childhood. Cancer 69:580-585

35. Pierga JY, Kalifa C, Terrier-Lacombe MJ, Habrand JL, Lemerle J (1993) Carcinoma of the choroid plexus: a pediatric experience. Med Pediatr Oncol 21:480-487

36. Pencalet P, Sainte-Rose C, Lellouch-Tubiana A, Kalifa C, Brunelle F, Sgouros S, Meyer P, Cinalli G, Zerah M, Pierre-Kahn A, Renier D (1998) Papillomas and carcinomas of the choroid plexus in children. J Neurosurg 88:521-528

37. McEvoy AW, Harding BN, Phipps KP, Ellison DW, Elsmore AJ, Thompson D, Harkness W, Hayward RD (2000) Management of choroid plexus tumours in children: 20 years experience at a single neurosurgical centre. Pediatr Neurosurg 32:192-199

38. Wolff JE, Sajedi M, Brant R, Coppes MJ, Egeler RM (2002) Choroid plexus tumours. Br J Cancer 87:1086-1091

39. Krishnan S, Brown PD, Scheithauer BW, Ebersold MJ, Hammack JE, Buckner JC (2004) Choroid plexus papillomas: a single institutional experience. J Neurooncol 68:49-55

40. Jeibmann A, Wrede B, Peters O, Wolff JE, Paulus W, Hasselblatt M (2007) Malignant progression in choroid plexus papillomas. J Neurosurg 107:199-202

41. Shintaku M, Nitta T, Matsubayashi K, Okamoto S (2008) Ossifying choroid plexus papilloma recurring with features of atypical papilloma. Neuropathology 28:160-164 
42. Berger C, Thiesse P, Lellouch-Tubiana A, Kalifa C, Pierre-Kahn A, Bouffet E (1998) Choroid plexus carcinomas in childhood: clinical features and prognostic factors. Neurosurgery 42:470-475

43. Chow E, Jenkins JJ, Burger PC, Reardon DA, Langston JW, Sanford RA, Heideman RL, Kun LE, Merchant TE (1999) Malignant evolution of choroid plexus papilloma. Pediatr Neurosurg 31:127-130

44. Maria BL, Graham ML, Strauss LC, Wharam MD (1985) Response of a recurrent choroid plexus tumor to combination chemotherapy. J Neurooncol 3:259-262

45. Valencak J, Dietrich W, Raderer M, Dieckmann K, Prayer D, Hainfellner JA, Marosi C (2000) Evidence of therapeutic efficacy of CCNU in recurrent choroid plexus papilloma. J Neurooncol 49:263-268

46. Pillai A, Rajeev K, Chandi S, Unnikrishnan M (2004) Intrinsic brainstem choroid plexus papilloma. Case report. J Neurosurg 100:1076-1078
47. Tacconi L, Delfini R, Cantore G (1996) Choroid plexus papillomas: consideration of a surgical series of 33 cases. Acta Neurochir (Wien) 138:802-810

48. Wolff JE, Sajedi M, Coppes MJ, Anderson RA, Egeler RM (1999) Radiation therapy and survival in choroid plexus carcinoma. Lancet 353:2126

49. Enomoto H, Mizuno M, Katsumata T, Doi T (1991) Intracranial metastasis of a choroid plexus papilloma originating in the cerebellopontine angle region: a case report. Surg Neurol 36:54-58

50. McEvoy AW, Galloway M, Revesz T, Kitchen ND (2002) Metastatic choroid plexus papilloma: a case report. J Neurooncol $56: 241-246$

51. Ortega-Martinez M, Cabezudo-Artero JM, Fernandez-Portales I, Pimentel JJ, Gomez de Tejada R (2007) Diffuse leptomeningeal seeding from benign choroid plexus papilloma. Acta Neurochir (Wien) 149:1229-1236 (discussion 1236-1227) 